\title{
Hamartoma adenomatoide epitelial respiratório: relato de caso
}

\author{
Respiratory epithelial adenomatoid hamartoma: case report
}

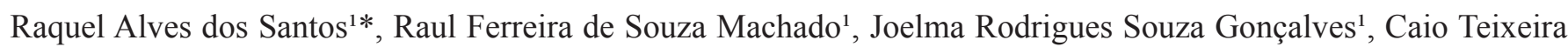
dos Santos ${ }^{1}$, Ivana Picone Borges de Aragão

Como citar esse artigo. dos Santos,

R.A; Machado, R.F.S; Gonçalves,

J.R.S; dos Santos, C.T; de Aragão, I.P.B. Hamartoma adenomatoide epitelial respiratório: relato de caso. Revista de Saúde.. 2021 Abr./Jul.; 12 (2): 28-32.

\begin{abstract}
Resumo
Os tumores benignos da cavidade nasal incluem um conjunto de lesões de caráter histopatológico heterogêneo. Dentre esses tumores, está incluso um subtipo de hamartoma, descrito como Hamartoma Adenomatoide Epitelial Respiratório (HAER) caracterizado por proliferações glandulares anormais derivadas da fossa nasal, nasofaringe e seios paranasais, principalmente do septo posterior, com alto potencial curativo, mas que constitui um diagnóstico desafiador na prática clínica devido a sua raridade. O objetivo foi relatar um caso de HAER, divulgar o conhecimento científico sobre essa tumoração benigna e rara, além de incentivar o diagnóstico diferencial. Paciente masculino, de 49 anos, com sintoma de dor facial intensa, de início agudo, de carácter contínuo, com intensidade 9 em 10 (sendo zero ausência de dor, e dez o máximo de dor), com irradiação para gengiva, nariz e orelha externa, agravada pela sensibilidade maior a temperatura mais baixa. Várias medidas diagnósticas e terapêuticas foram realizadas para o alívio da dor, mas sem sucesso. Procurou acompanhamento com diversas especialidades, que através de exames de imagem, foi evidenciado uma lesão tumoral em rinofaringe. Por suspeita de uma neoplasia maligna, foi realizado uma cirurgia para exérese total do tumor. Todavia, a análise anatomopatólogica confirmou o diagnóstico de HAER, uma tumoração benigna e rara. Pela raridade do HAER, com poucos relatos na literatura, é importante haver a divulgação, contribuindo para o conhecimento sobre a doença, possibilitando o diagnóstico diferencial naqueles com dor facial de forte intensidade, associado à presença de tumorações em nasofaringe ou paranasal.
\end{abstract}

Palavras-chave: Hamartoma; Dor facial; Obstrução nasal; Nasofaringe.

\begin{abstract}
Benign tumors of the nasal cavity include a set of heterogeneous histopathological lesions. Among these tumors is included a subtype of hamartoma, described as Hamartoma Adenomatoid Epithelial Respiratory (HAER) characterized by abnormal glandular proliferations derived from the nasal fossa, nasopharynx and paranasal sinuses, mainly from the posterior septum, with a highly curative potential, but which constitutes a challenging diagnosis in clinical practice due to its rarity. The objective was to report a case of HAER, disseminate scientific knowledge about this benign and rare tumor, in addition to encouraging differential diagnosis. Male patient, 49 years old, with severe acute facial pain symptom, of continuous character, with intensity 9 out of 10 (being zero absence of pain, and ten the maximum pain), with irradiation to the gums, nose and outer ear, aggravated by higher sensitivity at lower temperature. Several diagnostic and therapeutic measures were taken to relieve pain, but without success. He sought follow-up with several specialties, which through imaging exams showed a tumor lesion in the rhinopharynx. Due to the suspicion of a malignant neoplasm, surgery was performed for total excision of the tumor. However, anatomopathological analysis confirmed the diagnosis of HAER, a benign and rare tumor. Due to the rarity of HAER, with few reports in the literature, it is important to disseminate it, contributing to the knowledge about the disease, enabling the differential diagnosis in those with severe facial pain, associated with the presence of nasopharyngeal or paranasal tumors.
\end{abstract}

Keywords: Hamartoma; Facial pain; Nasal obstruction; Nasopharynx.

\section{Introdução}

Os tumores benignos da cavidade nasal incluem um conjunto de lesões de caráter histopatológico heterogêneo ${ }^{1}$, sendo uma condição pouco comum na prática clínica. Habitualmente, são assintomáticos, no entanto, quando os sintomas estão presentes demonstram comprometimento funcional do sistema do seio paranasal decorrente do crescimento significativo da lesãoº.
Dentre as tumorações benignas nasossinusais, estão incluídos os hamartomas, que são malformações compostas por um crescimento celular e tecidual de um órgão de maneira excessiva, desorganizada, porém autolimitada e localizada. De modo geral, podem se apresentar em qualquer parte do organismo, mas geralmente ocorrem no pulmão, fígado, rim e trato intestinal, mas raramente são encontrados no trato aerodigestivo superior ${ }^{3}$.

Um subtipo de hamartoma, é o descrito como

\footnotetext{
Afiliação dos autores:

${ }^{1}$ Discente da graduação do curso de medicina da Universidade de Vassouras, Vassouras, RJ, Brasil.

${ }^{2}$ Docente da graduação do curso de medicina da Universidade de Vassouras,, Vassouras, RJ, Brasil.
}

* Email de correspondência: raquelalvessantos.med@gmail.com 
hamartoma adenomatóide epitelial respiratório (HAER), o qual é caracterizado por proliferações glandulares anormais derivadas da fossa nasal, nasofaringe e seios paranasais, principalmente do septo posterior. ${ }^{3,4}$ Esse tipo de hamartoma, ainda com patogênese desconhecida ${ }^{5}$, é extremamente raro, tanto no Brasil quanto no mundo, e pode ser confundido com lesões mais agressivas, como papilomas invertidos ou adenocarcinoma do trato nasossinusal ${ }^{6}$, e até mesmo outras patologias como pólipo nasal inflamatório, resultando em intervenções inapropriadas para o diagnóstico.

Portanto, apresenta-se um relato de caso de HAER, a fim de demonstrar a relevância da identificação de tal doença como uma hipótese diagnóstica, uma vez que, detectada corretamente tem grande potencial curativo, modificando o bem-estar global do indivíduo.

\section{Material e Método}

Relato de caso, retrospectivo, de Hamartoma Adenomatoide Epitelial Respiratório, através da coleta dos dados clínicos (anamnese e exame físico) e exames laboratoriais evolutivamente, realizados com o próprio paciente, na forma de entrevista com os autores, após assinatura de termo de consentimento livre e esclarecido, garantindo o sigilo, o respeito à ética, privacidade e confidencialidade de todos os dados fornecidos. Revisão bibliográfica, usando descritores em Ciências da Saúde: "Hamartoma" "dor facial" "obstrução nasal" "nasofaringe", em base de dados científicos SciELO, PUBMED, no período dos últimos 25 anos, na língua português, inglês e espanhol. Os princípios éticos foram respeitados, a partir da submissão à apreciação e à aprovação do Comitê de Ética em Pesquisa da Universidade de Vassouras, número do parecer 4.339.956.

\section{Relato de Caso}

Paciente masculino, de 49 anos, em 26 de maio de 2019, iniciou um quadro agudo de dor facial, com início súbito, de carácter contínuo, com intensidade 9 em 10 (sendo zero ausência de dor, e dez o máximo de dor), com irradiação para gengiva, nariz e orelha externa, agravada pela sensibilidade maior a temperatura mais baixa, sem alívio com uso de medicação, não sabendo relatar qual utilizado, e acompanhado de uma pressão arterial variando de 100 x $80 \mathrm{mmHg}$ à 170 x $110 \mathrm{mmHg}$. Devido à intensidade da dor, procurou atendimento médico no mesmo dia, em uma unidade de pronto atendimento em saúde, onde foi medicado com antiinflamatório e analgésico intravenoso e, sendo liberado a seguir.

Porém, devido à persistência da dor e a associação com episódio de síncope, no mesmo dia 26, no período noturno, retornou ao serviço de emergência hospitalar, sendo internado para investigação.

Após avaliação médica, a principal hipótese diagnóstica foi de acidente vascular cerebral, sendo realizado exame de tomografia computadorizada (TC) de crânio, que revelou resultado normal.

Iniciado medicação analgésica para a dor facial, sendo prescrito, inicialmente, dipirona e oxigenioterapia, e, posteriormente, tramadol emorfina, houve persistência da dor, embora em caráter intermitente. Então, foi realizado uma punção de líquor, no qual o resultado foi normal, descartando a possibilidade de causa infecciosa. O paciente, foi liberado, após orientação para investigação com neurologista e receitado uso de carbamazepina. Relatou que a dor cedeu aos poucos com o uso dessa medicação.

Havia relato de passado de renite alérgica, dificuldade respiratória pelas narinas, roncos, bem como, episódios de epistaxe quando mais jovem, não sabendo relatar se bilateral ou unilateral.

Em 28/05/2019, procurou neurologista para investigação diagnóstica da dor, sendo solicitado uma angiorressonância arterial e venosa de crânio (angio$\mathrm{RM}$ ), e suspensa a carbamazepina.

$\mathrm{O}$ resultado da angio-RM revelou a presença de lesão expansiva de contornos irregulares, medindo $2.4 \mathrm{~cm}$ de diâmetro, com captação heterogênea do meio de contraste, havendo áreas de hipocaptação de permeio, localizada na cavidade nasal posteriormente e centralmente, junto a porção posterior do septo ósseo nasal, a qual determinava redução da coluna aérea da rinofaringe e, poderia corresponder a neoplasia.

Pela suspeita de neoplasia maligna, o paciente foi encaminhado, então, para otorrinolaringologia (ORL).

No mês de junho compareceu à consulta com o ORL, sendo realizado rinoscopia anterior, sem evidência de qualquer lesão. Foi solicitado um exame de videoendoscopia nasal, com laudo compatível com lesão expansiva vegetante, ligeiramente globosa na linha média da rinofaringe, com inserção aparentemente no teto da mesma, ocupando cerca de $70 \%$ da luz. Desvio septal à direita, obstrução nasal à direita e renite atópica. Encaminhado para procurar um cirurgião de cabeça e pescoço, para avaliação da possibilidade de tratamento cirúrgico.

Ainda no mês de junho compareceu à consulta com o cirurgião, sendo solicitado novo exame de TC de seios da face para melhor avaliação da extensão da doença, sendo verificado no laudo, a presença de lesão expansiva de aspecto pediculado/vegetante, indissociável da parede posterior da nasofaringe (figuras 1 e 2). Além de espessamento concêntrico e laminar da mucosa de revestimento de algumas células etmoidais bilateralmente e dos seios maxilares. 


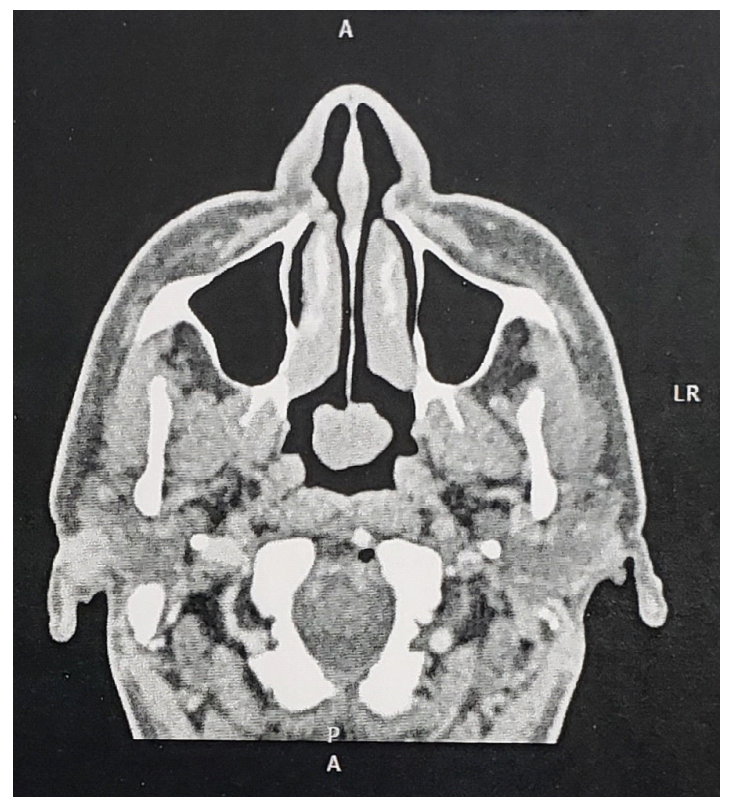

Figura 1. Corte axial de Tomografia Computadorizada de seios da face, mostrando lesão expansiva na parede posterior da nasofaringe.

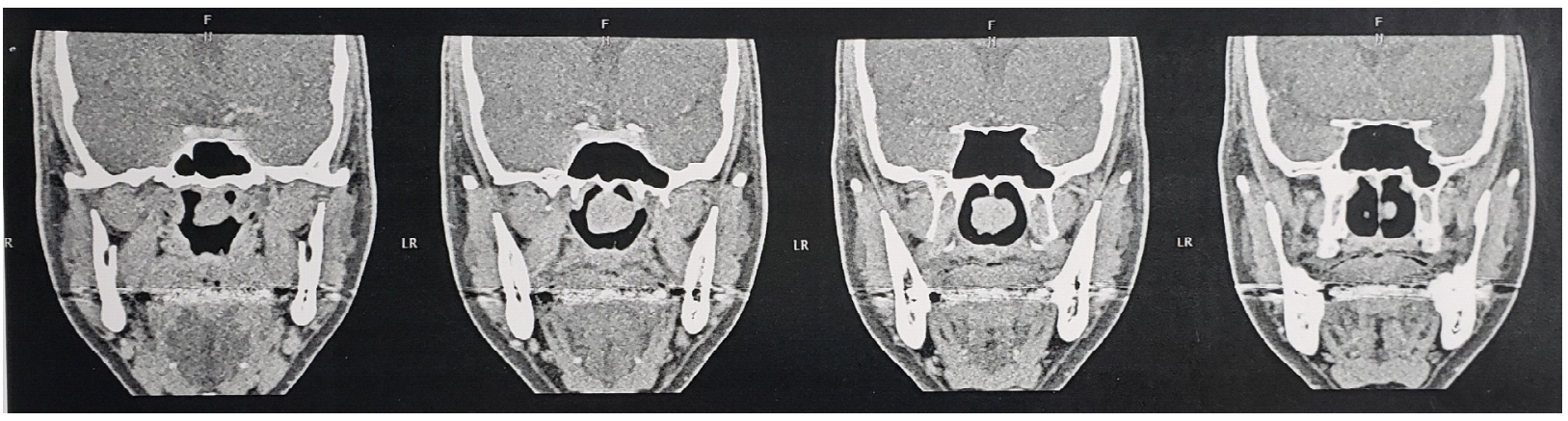

Figura 2. Corte coronal da Tomografia Computadorizada de seios da face mostrando lesão expansiva de aspecto pediculado/vegetante indissociável da parede posterior da nasofaringe.

Em 02/09/2019, foi submetido à cirurgia de exérese total do tumor da rinofaringe, sendo enviado dois fragmentos irregulares de tecido amarelo e elástico medindo em conjunto $1,0 \times 0,7 \times 0,3 \mathrm{~cm}$ para biópsia, cujo resultado anatomopatológico foi compatível com hamartoma adenomatoide epitelial respiratório.

No pós operatório, a recuperação do doente foi bem-sucedida, com boa cicatrização no local da cirurgia, houve melhora do olfato e desaparecimento da dor hemifacial. Foi solicitado uma TC de seios paranasais para controle pós-cirúrgico, que até o momento não havia sido realizada pelo paciente.

\section{Discussão}

Os hamartomas são entidades decorrentes de uma alteração no desenvolvimento celular e tecidual, podendo apresentar-se em qualquer parte do organismo, inclusive no epitélio superficial, glândulas seromucinosas, estroma fibroso ou vasos. ${ }^{7}$

Descrito pela primeira vez por Ozoleke Heffner em $1995^{\circ}$, um subtipo muito raro, o hamartoma adenomatoide epitelial respiratório (HAER), foi definido como uma lesão tumoral caracterizada por uma importante proliferação glandular revestida por epitélio respiratório ciliado originário do epitélio da superfície, com localização de maior ocorrência no septo nasal posterior $^{6}$ e com crescimento excessivo, desorganizado, mas autolimitado ${ }^{6,9,10}$.

Teorias para explicar a etiologia do HAER começam a ser discutidas em torno de uma explicação de natureza congênita ou secundária a um ambiente próinflmatório prolongado, mas que permanece com poucas evidências. ${ }^{4,8,9}$ Além disso, Ozolek \& Hunt, (2006) demonstraram que havia no HAER um alto número de 
desequilíbrios alélicos, e mesmo não sendo considerado um processo neoplásico, não exista evidências moleculares apoiando ou refutando essa possibilidade, sugerindo que o HAER possa ser uma neoplasia benigna e não um hamartoma. Ressaltando assim, a necessidade de estudos adicionais, mais aprofundados na análise biogenética dessas lesões.

Nas publicações de Miranda et al (2013) e Rodrigues, et al (2016), a maioria dos HAER ocorreu, predominantemente, em homens adultos, com uma proporção de homens para mulheres de 7: 1, com maior frequência entre a $3^{\mathrm{a}}$ e $9^{\mathrm{a}}$ décadas de vida.

No estudo de Wenig e Heffner (1995) foi identificado sintomatologia, predominantemente, de obstrução nasal, congestão nasal, epistaxe a rinossinusite crônica recorrente. No relato de Ingram et al (2006) a dor facial era o principal sintoma relacionado ao HAER, de forma semelhante ao presente relato. Duvillard \& Romanet (2008) demonstram que os hamartomas nasais podem ocasionar obstrução nasal e, consequentemente, ronco e epistaxe, e que quando volumosos podem causar importante dificuldade respiratória. ${ }^{11}$ Vira et al (2011) avaliou 54 pacientes com diagnóstico de HAER e observou que os sintomas presentes na maioria dos casos eram dor facial, pressão facial, congestão nasal, dores de cabeça e hiposmia. Da mesma forma, nosso caso demonstrou características semelhantes as citadas, como histórico de atopia nasal e epistaxe, possível hiposmia, obstrução nasal unilateral, dificuldade respiratória, além da importante dor facial, os quais corroborariam para a suspeita clínica do HAER.

Considerando que ainda possam existir dificuldades para o estabelecimento do diagnóstico do HAER, estudos demonstram a relevância do conhecimento prévio de outras patologias para diagnóstico diferencial, assim como, o estudo de achados nos exames de imagens que possam orientar na suspeita do diagnóstico correto e das medidas terapêuticas necessárias.

Jurado et al (2012) publicou que os principais diagnósticos diferenciais são os pólipos nasal e antrocoanal, o papiloma invertido e o adenocarcinoma de baixo grau ${ }^{4}$ (tabela 1 ).

No estudo de Vira et al (2011) foi observado que não havia características distintivas no exame endoscópico para sugerir um diagnóstico de HAER, bem como, não havia aparência característica ou específica na TC ou na ressonância magnética para ajudar a distinguir HAER de outra patologia nasossinusal, apenas imagens sugestivas de espessamento da mucosa ou mucoperiosteal, opacificação do seio, polipose nasal ou concha bolhosa, demonstrando que realmente HAER seja um achado incidental. ${ }^{7}$

De modo semelhante, nosso paciente apresentou nos exames de imagem, além da lesão em localização posterior do septo nasal, apenas o espessamento da mucosa, sendo o diagnóstico de HAER feito somente através da biópsia. E, assim como relatado na literatura, o HAER foi um achado incidental ${ }^{7,12}$ no presente relato.

Todavia Lima et al (2006) relata que o HAER aumenta significativamente a largura das fendas olfativas $^{13}$, assim também há relatos que outros autores descobriram que a ampliação da fenda olfativa maior

Tabela 1. Principais diagnósticos diferenciais e achados anatomopatológicos.

\begin{tabular}{|c|c|c|c|c|c|}
\hline & HAER & PAC & PN & PI & $A D C$ \\
\hline Origem & 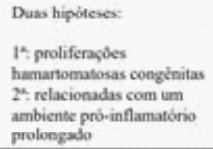 & $\begin{array}{l}\text { Escasso infiltrado } \\
\text { inflamatione e eccassa } \\
\text { cosiasfiliz. } \\
\end{array}$ & 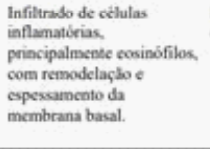 & $\begin{array}{l}\text { Epitelio excamoso } \\
\text { estratificado }\end{array}$ & $\begin{array}{l}\text { Pouce claro: no epitétio } \\
\text { superficial ou das } \\
\text { glinindulas seromucosas da } \\
\text { subenucosa. }\end{array}$ \\
\hline Localizacto & $\begin{array}{l}\text { Participasalo do septo naval } \\
\text { (emp particular a parte } \\
\text { posterior) }\end{array}$ & Scio maxilar & Scio etmoidal & Parede nasal lateral e secios & $\begin{array}{l}\text { Fossa nasal, seguido por } \\
\text { seios etmoodal e maxilar }\end{array}$ \\
\hline Achados IHQ & $\begin{array}{l}\text { Epitelio ciliado positivo } \\
\text { para CK } 7 \\
\text { Celulus de membrana basul } \\
\text { positivas para p63 e } \\
\text { HMWK }\end{array}$ & $\begin{array}{l}\text { Expressáo de MMP.2 } \\
\text { incrementada por celulus } \\
\text { inflamalórias }\end{array}$ & $\begin{array}{l}\text { Incremento da expressbo } \\
\text { de MMP.2 das celulas } \\
\text { inflamatórias }\end{array}$ & $\begin{array}{l}\text { Coexpressio de CK7. } \\
\text { CK8, CK19. p63 e HMWK } \\
\text { pelas celtulas escemosss } \\
\text { nilo queratinizadas de } \\
\text { transiçso }\end{array}$ & $\begin{array}{l}\text { Positivo para CK7, CK19 e } \\
\text { S100 } \\
\text { Negativo para CK20, } \\
\text { CDX2 }\end{array}$ \\
\hline $\begin{array}{l}\text { Caracteristicas } \\
\text { macrosépicas }\end{array}$ & $\begin{array}{l}\text { Massa polipoide ou } \\
\text { exofitica } \\
\text { Glandulss individuais } \\
\text { conectadas a superficie }\end{array}$ & $\begin{array}{l}\text { Muxoss odemaxiada do } \\
\text { seio maxilar, crescendo } \\
\text { atrowbs do meato mídio e } \\
\text { protusbo pela coana e } \\
\text { nasofaringe }\end{array}$ & $\begin{array}{l}\text { Edsma do tecido que surge } \\
\text { masis freyuentemente eas } \\
\text { fendas do meato medio e } \\
\text { poolappo na cavidade nasal }\end{array}$ & $\begin{array}{l}\text { Lenbes polipoides } \\
\text { Mucosa preservada }\end{array}$ & $\begin{array}{l}\text { Escasso tecido papilar oo } \\
\text { glandular }\end{array}$ \\
\hline $\begin{array}{l}\text { Caracteristicas } \\
\text { microsépicas }\end{array}$ & $\begin{array}{l}\text { Membrana basal espesss } \\
\text { Mutticamada de copiétio } \\
\text { respiratório ciliado } \\
\text { Protiferaçlo adenomatoide } \\
\text { Auséncia de um } \\
\text { componcnte glandulat } \\
\text { seromucoso }\end{array}$ & 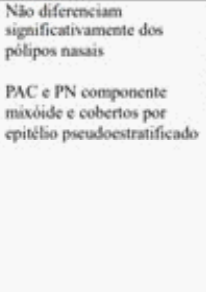 & $\begin{array}{l}\text { Pólipo edematcos, } \\
\text { eosinofilico, células } \\
\text { caliciformes, hiperplasia e } \\
\text { espessamemo da membrana } \\
\text { besal, com um infiltrado } \\
\text { cosinofilice. }\end{array}$ & $\begin{array}{l}\text { Endofitico, padrio de } \\
\text { creckimento invertido com } \\
\text { baixa extensalo estromal }\end{array}$ & 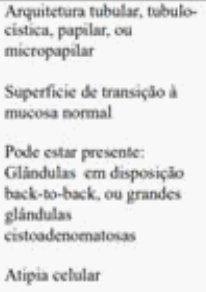 \\
\hline
\end{tabular}


que $10 \mathrm{~mm}$ na TC estão associados ao diagnóstico de $\mathrm{HAER}^{14}$, demonstrando que análise da fenda olfativa no exame de imagem pré-operatório pode se tornar uma maneira importante de avaliar a presença do HAER.

Mesmo antes do diagnóstico definitivo, no nosso caso, a conduta escolhida foi a exérese total do tumor a fim de que houvesse a análise anatomopatológica. Em concordância, a literatura demostra que por ser uma lesão benigna, com baixa recorrência, ou seja, com alto potencial curativo, o melhor tratamento para HAER é a ressecção cirúrgica com remoção completa ${ }^{3-7,9,10,13,14}$.

Além disso, Safi et al (2019) em sua revisão sistemática de artigos, descobriu também, que diversos autores citaram a melhora do olfato após a retirada do HEAR, assim como observado em nosso relato.

Todavia, vale ressaltar, que confundir o HAER com outras patologias mais graves pode levar a ressecções incompletas sem a devida margem de segurança, ou também, podem ser adotadas medidas terapêuticas mais invasivas sem a real necessidade, afetando, assim, a qualidade de vida do paciente.

\section{Conclusão}

O hamartoma adenomatoide epitelial respiratório constitui um diagnóstico desafiador para muitos médicos, uma vez que, é uma lesão rara, com variada apresentação e, que pode ser confundida com outras patologias, comprometendo a escolha terapêutica adequada.

Pela raridade do tumor, com poucos relatos na literatura, é importante haver notificação, contribuindo para o melhor conhecimento e avaliação pré-operatória, além de aumentar a possibilidade do diagnóstico diferencial, naqueles com dor facial intensa, obstrução nasal e tumoração nasofaríngea ou paranasal.

\section{Referências}

1. Larangeio J, Barros F, Vaz R, Cardoso V, Santos M. Tumores benignos nasosinusais: Estudo retrospectivo. Rev Port Otorrinol Cir Cabeça e Pescoço 2012; 50(2):129-135

2. Harvey RJ, Sheahan PO, Schlosser RJ. Surgical Management of Benign Sinonasal Masses. Otolaryngologic Clin North Am 2009; 42(2);353-375.

3. Cao Z, Gu Z, Yang J, Jin M. Respiratory epithelial adenomatoid hamartoma of bilateral olfactory clefts associated with nasal polyposis: Three cases report and literature review. Auris Nasus Larynx 2010; 37(3):352-356.

4. Jurado FXA, et al. Importancia del diagnóstico diferencial en rinología: hamartoma respiratorio adenoepitelial. Acta Otorrinolaringol Esp 2012; 63(1):55-61.

5. Muñoz AT, Cunchillos MN, Simal MG, Campillo AG. Hamartoma Nasal. Acta Otorrinolaringol Esp 2010; 61(2):163-165.

6. Wenig BM, Heffner CDK. Respiratory Epithelial Adenomatoid Hamartomas of the Sinonasal Tract and Nasopharynx: A Clinicopathologic Study of 31 Cases. Annals Otol Rhinol Laryngol 1995; 104:639-645.

7. Vira D, Bhuta S, Wang MB. Respiratory epithelial adenomatoid hamartomas. Laryngoscope 2011;121(12):2706-9.
8. Ozolek JA, Hunt JL. Tumor suppressor gene alterations in respiratory epithelial adenomatoid hamartoma (REAH): comparison to sinonasal adenocarcinoma and inflamed sinonasal mucosa. Am J Surg Pathol 2006; 30(12):1576-80

9. Liang J, O’Malley BW, Feldman M, Newman JG. A case of respiratory epithelial adenomatoid hamartoma. Am J Otolaryngol 2007;28(4):277-279.

10. Lee JT, Garg R, Brunworth J, Keschner DB, Thompson LD. Sinonasal respiratory epithelial adenomatoid hamartomas: series of 51 cases and literature review. Am J Rhinol Allergy 2013;27(4):322-8

11. Duvillard C, Romanet P. Tumori benigni della faringe. EMC Otorinolaringoiatria 2008;7(2):1-7.

12. Endo R, Matsuda H, Takahashi M, Hara M, Inaba H, Tsukuda M. Respiratory epithelial adenomatoid hamartoma in the nasal cavity. Acta Otolaryngol 2002;122(4):398-400.

13. Lima NB, Jankowski R, Georgel T, et al. Respiratory adenomatoid hamartoma must be suspected on CT-scan enlargement of the olfactory clefts. Rhinology 2006;44(4): 264-269.

14. Safi, C., Li, C., Tabaee, A., Ramakrishna, R., \& Riley, C. A. Outcomes and imaging findings of respiratory epithelial adenomatoid hamartoma: a systematic review. Int For Allergy Rhinol 2019;9(6):674-680. 